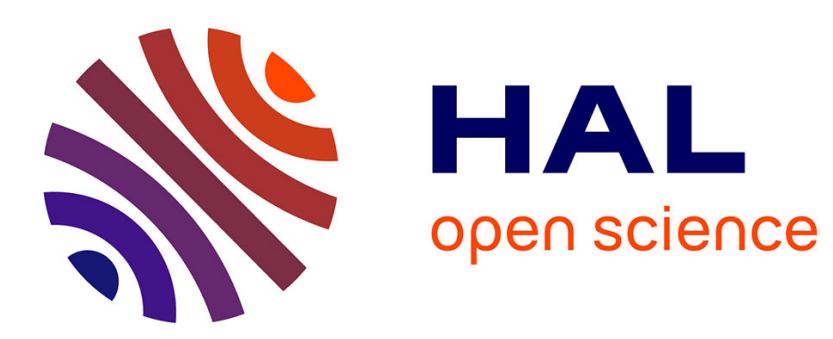

\title{
Existence and non-existence of global solutions to initial boundary value problems for nonlinear evolution equations with the strong dissipation
}

Akisato Kubo, Jean-Pierre Lohéac

\section{- To cite this version:}

Akisato Kubo, Jean-Pierre Lohéac. Existence and non-existence of global solutions to initial boundary value problems for nonlinear evolution equations with the strong dissipation. 2009. hal-00361333

\section{HAL Id: hal-00361333 \\ https://hal.science/hal-00361333}

Preprint submitted on 15 Feb 2009

HAL is a multi-disciplinary open access archive for the deposit and dissemination of scientific research documents, whether they are published or not. The documents may come from teaching and research institutions in France or abroad, or from public or private research centers.
L'archive ouverte pluridisciplinaire HAL, est destinée au dépôt et à la diffusion de documents scientifiques de niveau recherche, publiés ou non, émanant des établissements d'enseignement et de recherche français ou étrangers, des laboratoires publics ou privés. 


\title{
Existence and Non-existence of Global Solutions to Initial Boundary Value Problems for Nonlinear Evolution Equations with the Strong Dissipation
}

\author{
Akisato Kubo $^{\mathrm{a}}$, Jean-Pierre Lohéac ${ }^{\mathrm{b}}$ \\ ${ }^{a}$ Department of Mathematics, School of Health Sciences, Fujita Health University, \\ Toyoaake, Aichi, 470-1192, JAPAN, \\ akikubo@fujita-hu.ac.jp \\ ${ }^{b}$ Université de Lyon, École Centrale de Lyon, Département Mathématiques Informatique, \\ Institut Camille-Jordan (C.N.R.S. U.M.R. 5208), 36 avenue Guy-de-Collongue, 69134 \\ ÉCULLY Cedex, FRANCE, \\ Jean-Pierre.Loheac@ec-lyon.fr, \\ Independent Moscow University, Laboratoire J.-V. Poncelet (C.N.R.S. U.M.I. 2615), \\ Bol. Vlasyevsky Per. 11, 119002 MOSCOW, RUSSIA, \\ Jean-Pierre.Loheac@math.cnrs.fr
}

\begin{abstract}
The main purpose in this paper is to investigate existence and non-existence of global solutions of the initial Dirichlet-boundary value problem for evolution equations with the strong dissipation. Many authors studied classes consisting of such type of equations for which initial boundary value problems possess global solutions. For this purpose we consider a related problem and seek global solutions and blow-up solutions of it depending on whether it belongs to such classes or not.
\end{abstract}

Key words: evolution equation, strong dissipation, blow-up, global existence

2000 MSC: 35L70, 35L15, 35L20, 58K55

\section{Introduction}

In this paper our main concern is the initial Dirichelt-boundary value problem for a nonlinear evolution equation with the strong dissipation with respect to $v:=v(x, t)$ : 


$$
(D B) \begin{cases}v_{t t}-\lambda v_{x x t}-d v_{x x}=A\left(v_{x}\right) v_{x x} & \text { in }(0, \pi) \times(0, T) \\ v(0, t)=0, \quad v(\pi, t)=0 & \text { on }(0, T) \\ v(x, 0)=h_{0}(x), \quad v_{t}(x, 0)=h_{1}(x) & \text { in }(0, \pi)\end{cases}
$$

where the nonlinear term is of the form:

$$
A\left(v_{x}\right)=a v_{x}+\sigma\left(v_{x}\right)
$$

and $\lambda>0, a \neq 0$ and $d \neq 0$ are constants and $\sigma\left(v_{x}\right)$ will be specified in section 2 . The sign of $d$ plays a very important role to discuss the existence of global in time solutions to $(D B)$. In fact, if $d<0$, we obtain not only blow-up solutions but also global solutions to $(D B)$ depending on the choice of initial data. If $d>0$, it will be shown that for any intial data, there exist global solutions such that they decay exponentially as $t \rightarrow \infty$. Hence initial data of the solutions to $(D B)$ will be classified from this standpoint in section 2 .

There is a number of papers of the initial-Dirichlet boundary value problem for the type of the equation (1.1). Greenberg [5] considered the intial Dirichlet boundary value problem:

$(G)\left\{\begin{array}{l}\rho_{0} u_{t t}=E\left(u_{x}\right) u_{x x}+\lambda u_{x x t}, \quad(x, t) \in(0,1) \times(0, T) \\ u(x, 0)=x, u_{t}(x, 0)=V^{0}(x) \\ u(0, t)=0, u(1, t)=1\end{array}\right.$

where $\rho_{0}$ and $\lambda$ are positive constants. He proved the existence of the generalized solution to the problem in $(0,1) \times[0, T]$ for each $T>0$ provided that in $[0,1] \times \bar{R}_{+}$

$$
E\left(u_{x}\right)=\int_{1}^{u_{x}} e(\xi) d \xi, \quad E\left(u_{x}\right)>0 .
$$

(1.5) describes a local statement of balance of momentum for materials for which the stress is related to strain rate through some constitutive equation(cf [6]). In a more general situation M.Tsutumi [13] treated with the problem on some Hilbert spaces. Kakita [7] obtained the existence of weak periodic solution for the type of the equation (1.5). Since then, many authors have 
investigated the global existence as well as the asymptotic behavior of solutions to the type of the problem $(G)$ under assumptions analogous to (1.6)(see Ebihara [2]-[4], Yamada [15], Kawashima-Shibata [8] and Kobayashi-PecherShibata [9] and the referrences cited in these papers). Ebihara [3] considered the following problem :

$$
(E)\left\{\begin{array}{l}
u_{t t}-\Delta u_{t}=F\left(x, t, u, u_{x_{i}}, u_{x_{i} x_{j}}, u_{t}, u_{t x_{i}}\right), \quad(x, t) \in \Omega \times[0, \infty) \\
u(x, 0)=u_{0}(x), \quad u_{t}(x, 0)=u_{1}(x), \\
\left.u\right|_{\partial \Omega}=\varphi(x)
\end{array}\right.
$$

where $\Omega$ is a bounded domain in $R^{n}$ and (1.7) is of a much more general form than $(1.1)(\operatorname{cf}[2][4])$. He proved the local in time existence of solutions to $(E)$ in [3]. In fact, in section 2 we will find blow-up solutions of $(D B)$, which is a special case of $(E)$. Ebihara [3] also considered a restricted case of $(E)$ :

$(E)^{\prime}\left\{\begin{array}{l}u_{t t}-\Delta u_{t}=P(\nabla u) \Delta u, \\ u(x, 0)=u_{0}(x), u_{t}(x, 0)=u_{1}(x), \\ u(\xi, t)=S(\xi), \quad \xi \in \partial \Omega\end{array}\right.$

under the condition

$$
P(0, \cdots, 0)=0, \quad P\left(a_{1}, \cdots, a_{n}\right) \equiv \delta>0
$$

where $P(\nabla u)$ is a polynomial in $u_{x_{i}}(i=1,2, \cdots, n)$ and $S(\xi)=\sum_{j=1}^{n} a_{j} \xi_{j}\left(a_{j}\right.$ :constant). He proved the existence of the global solution by using a transformation $u(x, t)=e^{-\delta t} v(x, t)+S(x)$. It can be observed that $(E)^{\prime}$ is a general case of $(G)$, too.

In [3] and [5], as stated above, the conditions (1.6) and (1.8) are required to obtain existence theorems of global solutions respectively. In order to discuss non-existence of global solutions of $(G)$ and $(E)^{\prime}$ if such conditions are not satisfied we consider a related problem as

$$
(G E) \begin{cases}u_{t t}-\lambda u_{x x t}=\left(a u_{x}+\sigma\left(u_{x}-1\right)\right) u_{x x} & \text { in }(0, \pi) \times(0, T) \\ u(0, t)=0, \quad u(\pi, t)=\pi . & \text { on }(0, T) \\ u(x, 0)=x, \quad u_{t}(x, 0)=V^{0}(x) . & \text { in }(0, \pi)\end{cases}
$$


Putting $u(x, t)=x+v(x, t),(G E)$ is reduced to $(G E)^{\prime}\left\{\begin{array}{l}v_{t t}-\lambda v_{x x t}-a v_{x x}=\left(a v_{x}+\sigma\left(v_{x}\right)\right) v_{x x} \\ v(0, t)=0, \quad v(\pi, t)=0 \\ v(x, 0)=0, \quad v_{t}(x, 0)=V^{0}(x)\end{array}\right.$

which is the type of the problem $(D B)$.

Recently in [12] Othmer and Stevens derived a parabolic-ODE system modeling reinforced random walk proposed by Davis [1], which is applied to chemotactic aggregation of myxobacteria etc.. Mathematical analysis of this model was done by Levine and Sleeman [10](see also Sleeman and Levine [11]). They consider the following problem arising from the parabolic-ODE proposed by Othmer and Stevens:

$(C) \begin{cases}u_{t t}-D \Delta u_{t}-d D \nabla \cdot\left(u_{t} \nabla u\right)=0 & \text { in } \Omega \times(0, T) \\ \left.\partial_{\nu} u\right|_{\partial \Omega}=0 & \text { on } \partial \Omega \times(0, T) \\ u(x, 0)=u_{0}(x), \quad u_{t}(x, 0)=u_{1}(x) & \text { in } \Omega .\end{cases}$

where $D$ is a positive constant and $d$ is a real number. When $n=1$ and $D=1$, they construct both global and blow-up in finite time solutions to $(C)$ for $d=1$ and -1 respectively. Yan-Chen-Liu [14] extended their result by improving their method. Making use of Levine and Sleeman's way we will seek both global and blow-up solutions to $(D B)$.

The main purpose in this paper is to investigate existence and non- existence of global solutions to $(D B)$ and properties of solutions. In section 2 we will construct blow-up in finite time solutions to $(D B)$ for $d<0$, and global solutions for $d>0$. In subsection 2.3, comparing our result to known results [3] and [5] we discuss the relationship between existence and non-existence of global solutions of $(G E)$ and the property of the non-linear term. 


\section{Existence and non-existence of global solutions}

\subsection{Construction of solutions}

By an analogous manner to the way used in Levine and Sleeman [10], we will construct blow-up solutions and global solutions to $(D B)$. In fact we will seek the solution to $(D B)$ in the form of

$$
v(x, t)=\sum_{n=1}^{\infty} a_{n} \exp (c N n t) \sin (N n x), \quad(x, t) \in(0, \pi) \times(0, T)
$$

where $a_{n}$ and $c$ are suitably chosen in the following lemma and $N$ is a positive integer. We sometimes use the following notations

$$
E_{n}(t)=\exp (n c N t), S_{n}(x)=\sin (n N x) \text { and } C_{n}(x)=\cos (n N x)
$$

for our convenience.

Lemma 1. Put $a_{n}=-4 \lambda c \varepsilon^{n} /(a n)$. Then

$$
v(x, t)=\frac{-4 \lambda c}{a} \sum_{n=1}^{\infty} \frac{\varepsilon^{n} \exp (c N n t)}{n} \sin (N n x)
$$

satisfies

$v_{t t}-\lambda v_{x x t}-d v_{x x}-a v_{x} v_{x x}=\frac{4 c^{2} \lambda^{2} N^{3}}{a} \sum_{n=2}^{\infty} \varepsilon^{n} \exp (c N n t) \sum_{k+l=n} k \sin (N(k-l) x)$

in a formal sense where $\varepsilon$ is a parameter and $c$ is a solution of an indicial equation $c^{2}+\lambda N c+d=0$.

Proof. Substituting $v(x, t)$ of the form $\sum_{n=1}^{I} a_{n} \exp (c N n t) \sin (N n x)$ into (1.1) we have for an integer $I \geq 2$

$v_{t t}-d v_{x x}-\lambda v_{t x x}=N^{2} \sum_{n=1}^{I} a_{n} n^{2}\left(c^{2}+\lambda N n c+d\right) \exp (c N n t) \sin (N n x)$

and in the right hand side of (1.1)

$$
a v_{x} v_{x x}=-a N^{3} \sum_{n=2}^{I} \sum_{k+l=n} k^{2} a_{k} l a_{l} \exp (c N(k+l) t) \sin (N k x) \cos (N l x)
$$




$$
\begin{aligned}
= & \frac{-a}{2} N^{3} \sum_{n=2}^{I} \sum_{k+l=n} k^{2} a_{k} l a_{l} \exp (c N(k+l) t) \cdot(\sin (N(k+l) x)+\sin (N(k-l))) \\
= & \frac{-a}{2} N^{3} \sum_{n=2}^{I} \exp (c N n t) \sin (N n x) \sum_{k=1}^{n-1} k^{2} a_{k}(n-k) a_{n-k} \\
& -\frac{a}{2} N^{3} \sum_{n=2}^{I} \exp (c N n t) \sum_{k+l=n} k^{2} a_{k} l a_{l} \sin (N(k-l) x) .
\end{aligned}
$$

In the case of $n=1$, for any $a_{1}$ we determine $c$ so that it satisfies

$$
c^{2}+\lambda N c+d=0 .
$$

Then taking account of (2.3) and (2.4) we determine $a_{n}, n=2,3, \cdots, I$ so that it holds

$$
n^{2}\left(c^{2}+N \lambda n c+d\right) a_{n}=-\frac{a}{2} N \sum_{k=1}^{n-1} k^{2}(n-k) a_{k} a_{n-k}
$$

equivalently

$$
4 n \lambda c a_{n}=\frac{-2 a}{n(n-1)} \sum_{k=1}^{n-1} k\left(k a_{k}\right)\left((n-k) a_{n-k}\right) .
$$

For

$$
a_{n}=-4 \lambda c \varepsilon^{n} /(a n),
$$

the right hand side of (2.7) gives

$$
\begin{gathered}
-\frac{2 \lambda^{2}}{n(n-1) a} \sum_{k=1}^{n-1} k \cdot 4 c \varepsilon^{k} \cdot 4 c \varepsilon^{n-k} \\
=-\frac{32 c^{2} \lambda^{2}}{n(n-1) a} \varepsilon^{n} \sum_{k=1}^{n-1} k=-\frac{16}{a} c^{2} \lambda^{2} \varepsilon^{n}
\end{gathered}
$$

which is equal to the left hand side of (2.7). Thus putting

$$
v_{I}(x, t)=4 \sum_{n=1}^{I} \frac{-\lambda c}{n a} \varepsilon^{n} E_{n}(t) S_{n}(x)
$$


we obtain the following equality in a formal sense

$$
v_{I t t}-\lambda v_{I x x t}-d v_{I x x}-a v_{I x} v_{I x x}=\frac{4 N^{3} c^{2} \lambda^{2}}{a} \sum_{n=2}^{I} \varepsilon^{n} E_{n}(t) \sum_{k+l=n} k S_{k-l}(x) .
$$

Letting $I \rightarrow \infty$ in (2.9), we obtain (2.2). Hence the proof is complete.

Next we further represent the right hand side of (2.2) in a explicit form.

Lemma 2. Under the same assumption as in lemma 1 and $|\varepsilon| \exp (c N t)<$ 1, we see that the right hand side of (2.2):

$$
\frac{4 c^{2} \lambda^{2} N^{3}}{a} \sum_{n=2}^{\infty} \sum_{k+l=n} k \varepsilon^{n} \exp (N n c t) \sin (N(k-l) x)=-\frac{N \lambda c(\varepsilon \exp (N c t))^{2}}{1-(\varepsilon \exp (N c t))^{2}} v_{x x}
$$

in a formal sense, that is, (2.2) is rewritten by

$$
v_{t t}-\lambda v_{x x t}-d v_{x x}-a v_{x} v_{x x}=-\frac{N \lambda c(\varepsilon \exp (N c t))^{2}}{1-(\varepsilon \exp (N c t))^{2}} v_{x x} .
$$

Proof. The proof is consist of two parts.

i) In the case of $n=2 i+1, i=1,2, \cdots,(I-1) / 2$, we have for $k+l=2 i+1$

$$
\begin{gathered}
\sum_{k=1}^{2 i} k S_{k-l}=\sum_{k=i+1}^{2 i} k S_{k-l}-\sum_{l=i+1}^{2 i} k S_{l-k} \\
=\sum_{k=i+1}^{2 i}(k-l) S_{k-l}=\sum_{k=i+1}^{2 i}(2 k-2 i-1) S_{2 k-2 i-1}
\end{gathered}
$$

putting $k=i+j$

$$
=\sum_{j=1}^{i}(2 j-1) S_{2 j-1}
$$

We get in the right hand side of $(2.9)$ for $k+l=2 i+1$ :

$$
\begin{array}{r}
\frac{4 N^{3} \lambda^{2} c^{2}}{a} \sum_{k=1}^{2 i} \varepsilon^{n} E_{n}(t) k S_{k-l} \\
=\frac{4 N^{3} \lambda^{2} c^{2}}{a} \sum_{j=1}^{i} \varepsilon^{2 i+1} E_{2 i+1}(t)(2 j-1) S_{2 j-1}(x) .
\end{array}
$$


Therefore (2.11) yields that the right hand side of (2.9):

$$
\begin{gathered}
\frac{4 N^{3} \lambda^{2} c^{2}}{a} \sum_{i=1}^{(I-1) / 2} \sum_{j=1}^{i} \varepsilon^{2 i+1} E_{2 i+1}(t)(2 j-1) S_{2 j-1}(x) \\
=\frac{4 N^{3} \lambda^{2} c^{2}}{a} \sum_{j=1}^{(I-1) / 2} \varepsilon^{2 j+1} E_{2 j+1}(t)(2 j-1) S_{2 j-1}(x) \sum_{s=0}^{(I-1) / 2-j}\left(e^{2 c N t} \varepsilon^{2}\right)^{s} \\
=\sum_{j=1}^{(I-1) / 2} g_{2 j-1}(t)(2 j-1) S_{2 j-1}(x)
\end{gathered}
$$

where

$g_{2 j-1}(t)=\frac{4 N^{3} \lambda^{2} c^{2}\left(\varepsilon E_{1}(t)\right)^{2}}{a} \varepsilon^{2 j-1} E_{2 j-1}(t)\left(1+e^{2 c N t} \varepsilon^{2}+\cdots+\left(e^{2 c N t} \varepsilon^{2}\right)^{(I-1) / 2-j}\right)$.

ii) In the case of $n=2 i, i=2, \cdots, I / 2$ we have in the same way as in i) for $k+l=2 i$

$$
\sum_{k=1}^{2 i-1} k S_{k-l}(x)=\sum_{j=1}^{i-1} 2 j S_{2 j} .
$$

Therefore we get the right hand side of (2.9):

$$
\begin{gathered}
\frac{4 N^{3} \lambda^{2} c^{2}}{a} \sum_{i=2}^{I / 2} \sum_{j=1}^{i-1} \varepsilon^{2 i} E_{2 i}(t)(2 j) S_{2 j}(x) \\
=\frac{4 N^{3} \lambda^{2} c^{2}}{a} \sum_{j=1}^{I / 2-1} \varepsilon^{2 j+2} E_{2 j+2}(t)(2 j) S_{2 j}(x)\left(1+e^{2 c N t} \varepsilon^{2}+\cdots+\left(e^{2 c N t} \varepsilon^{2}\right)^{I / 2-1-j}\right) \\
=\sum_{j=1}^{I / 2-1} g_{2 j}(t)(2 j) S_{2 j}(x)
\end{gathered}
$$

where

$$
g_{2 j}(t)=\frac{4 N^{3} \lambda^{2} c^{2}\left(\varepsilon E_{1}(t)\right)^{2}}{a} \varepsilon^{2 j} E_{2 j}(t)\left(1+e^{2 c N t} \varepsilon^{2}+\cdots+\left(e^{2 c N t} \varepsilon^{2}\right)^{I / 2-1-j}\right) .
$$


Consequently we obtain by combining (2.12) with (2.14)

$$
\text { the right hand side of }(2.9)=\sum_{n=1}^{I-2} g_{n}(t) n S_{n}(x) \text {. }
$$

Since taking $I \rightarrow \infty$ in $(2.15)$ we have for $\left|\varepsilon E_{1}(t)\right|<1$

$$
\begin{aligned}
g_{n}(t) n S_{n}(x)= & \frac{4 N^{3} \lambda^{2} c^{2}\left(\varepsilon E_{1}(t)\right)^{2}}{a} \varepsilon^{n} E_{n}(t)\left(1+\left(\varepsilon e^{c N t}\right)^{2}+\left(\varepsilon e^{c N t}\right)^{4}+\cdots\right) n S_{n}(x) \\
= & \frac{4 N^{3} \lambda^{2} c^{2}\left(\varepsilon E_{1}(t)\right)^{2}}{a} \varepsilon^{n} E_{n}(t) \frac{1}{1-e^{2 c N t} \varepsilon^{2}} n S_{n}(x),
\end{aligned}
$$

as $I \rightarrow \infty$ we see

$$
\begin{aligned}
& \text { the right hand side of }(2.9)=\frac{N \lambda c\left(\varepsilon E_{1}(t)\right)^{2}}{1-\left(e^{c N t} \varepsilon\right)^{2}} \sum_{n=1}^{\infty} \frac{4 c \lambda}{a} \varepsilon^{n} E_{n}(t) N^{2} n S_{n}(x) \\
& =-\frac{N \lambda c\left(\varepsilon E_{1}(t)\right)^{2}}{1-\left(\varepsilon E_{1}(t)\right)^{2}} v_{x x}
\end{aligned}
$$

Hence it is seen that $v(x, t)$ satisfies in a formal sense

$$
v_{t t}-\lambda v_{x x t}-d v_{x x}-\left(a v_{x}-\frac{N \lambda c\left(\varepsilon E_{1}(t)\right)^{2}}{1-\left(E_{1}(t) \varepsilon\right)^{2}}\right) v_{x x}=0
$$

which completes the proof.

Let $x_{0} \in[0, \pi]$ be such that $\cos \left(N x_{0}\right)=\operatorname{sgn}(\varepsilon)$ and let us define

$$
\sigma\left(v_{x}\right)(t)=-\frac{\left(a v_{x}\left(x_{0}, t\right)\right)^{2}}{16 N c \lambda-8 a v_{x}\left(x_{0}, t\right)}
$$

In the following lemma, we give explicit expressions of $v(x, t), v_{x}(x, t)$ and $v_{t}(x, t)$.

Lemma 3. i) Under the same assumptions as in lemma $1 v(x, t)$ and its first derivatives with respect to $x$ and $t$ converge for $|\varepsilon|<1$ and $(x, t) \in$ $(0, \pi) \times[0, T(\varepsilon, N))$. They can be written as follows,

$$
v_{x}(x, t)=\frac{-4 c \lambda N}{a} \varepsilon E_{1}(t) \frac{C_{1}(x)-\varepsilon E_{1}(t)}{1-2 \varepsilon E_{1}(t) C_{1}(x)+\left(\varepsilon E_{1}(t)\right)^{2}},
$$




$$
v_{t}(x, t)=\frac{-4 c^{2} \lambda N}{a} \varepsilon E_{1}(t) \frac{S_{1}(x)}{1-2 \varepsilon E_{1}(t) C_{1}(x)+\left(\varepsilon E_{1}(t)\right)^{2}},
$$

and

$$
v(x, t)=\frac{-4 \lambda c}{a} \arctan \left(\frac{\varepsilon \exp (c N t) \sin (N x)}{1-\varepsilon \exp (c N t) \cos (N x)}\right)
$$

where $T(\varepsilon, N)=-\log |\varepsilon| /(N c)$ in the case of $c>0$ and $T(\varepsilon, N)=\infty$ in the case of $c<0$.

ii) We have the following expression of the coefficient of the right hand side of $(2.10)$

$$
-c \lambda N \frac{(\varepsilon \exp (c N t))^{2}}{1-(\varepsilon \exp (c N t))^{2}}=\sigma\left(v_{x}\right)(t) .
$$

Proof. i) It is easily seen that for $0 \leq t<T(\varepsilon, N)$ and $0<|\varepsilon|<1(2.1)$ and its first derivatives converge absolutely in $[0, \pi] \times[0, T)$. It is well known that

$$
\sum_{n=1}^{\infty} \frac{a^{n}}{n} \sin (n x)=\arctan \left(\frac{a \sin x}{1-a \cos x}\right),-\pi<x<\pi, \quad|a|<1 .
$$

Due to the above expression we obtain

$$
\begin{aligned}
& v(x, t)=\frac{-4 \lambda c}{a} \sum_{n=1}^{\infty} \frac{\varepsilon^{n} \exp (c N n t)}{n} \sin (N n x) \\
= & \frac{-4 \lambda c}{a} \arctan \left(\frac{\varepsilon \exp (c N t) \sin (N x)}{1-\varepsilon \exp (c N t) \cos (N x)}\right),
\end{aligned}
$$

which gives the explicit expressions of $v_{x}$ and $v_{t}$ by a simple calculation. Hence we arrive at (2.18)-(2.20).

ii) It is seen by (2.18) that for $x_{0}$ satisfying $\cos \left(N x_{0}\right)=\operatorname{sgn}(\varepsilon)$

$$
\begin{aligned}
& v_{x}\left(x_{0}, t\right)=\frac{-4}{a} N c \lambda|\varepsilon| \exp (N c t) \frac{1-|\varepsilon| \exp (N c t)}{1-2|\varepsilon| \exp (N c t)+|\varepsilon|^{2} \exp (2 N c t)} \\
= & \frac{-4}{a} N c \lambda|\varepsilon| \exp (N c t) \frac{1}{1-|\varepsilon| \exp (N c t)}, \quad \text { in }[0, T(\varepsilon, N)) .
\end{aligned}
$$

Then, since by (2.21) we easily see that $v_{x}\left(x_{0}, t\right) \neq 4 a^{-1} N c \lambda$ for any $t \in$ $[0, T(\varepsilon, N)),(2.21)$ also yields that

$$
|\varepsilon| \exp (N c t)=\frac{v_{x}\left(x_{0}, t\right)}{-4 a^{-1} N c \lambda+v_{x}\left(x_{0}, t\right)} .
$$


By using (2.22) we see

$$
\frac{N \lambda c(\varepsilon \exp (N c t))^{2}}{1-(\varepsilon \exp (N c t))^{2}}=\frac{v_{x}^{2}\left(x_{0}, t\right)}{\frac{16}{a^{2}} N c \lambda-\frac{8}{a} v_{x}\left(x_{0}, t\right)}
$$

Hence we obtain ii) of lemma 3.

Remark. Note that by (2.21) and (2.23) we have for $a<0(>0)$

$$
v_{x}\left(x_{0}, t\right)>0 \text { and } \sigma\left(v_{x}\right)<0
$$

in the case of $c>0$ and

$$
v_{x}\left(x_{0}, t\right)<0(>0) \text { and } \sigma\left(v_{x}\right)>0
$$

in the case of $c<0$ respectively. Namely, $v_{x}\left(x_{0}, t\right)$ and $c / a$ have opposite signe, as well as $\sigma\left(v_{x}\right)$ and $c$.

From lemma 3 and (2.10), we easily deduce

$$
v_{t t}-\lambda v_{x x t}-d v_{x x}-a v_{x}=-\sigma\left(v_{x}\right) v_{x x}, \quad \text { in }(0, \pi) \times[0, T(\varepsilon, N))
$$

in a formal sense and it will be justified in the next subsection.

\subsection{Existence and non-existence of global solutions to (DB)}

We denote the solutions of (2.6) by

$$
c_{1}=\frac{-\lambda N+\sqrt{\lambda^{2} N^{2}-4 d}}{2}, \quad c_{2}=\frac{-\lambda N-\sqrt{\lambda^{2} N^{2}-4 d}}{2}
$$

and $c_{i}$ is further denoted by $c_{i}^{-}$if $d<0$ and $c_{i}^{+}$if $d>0$ for $i=1,2$, respectively. It is easily seen that $c_{1}^{-}>0$ and $c_{2}^{-}, c_{1}^{+}, c_{2}^{+}<0$. Denote initial data of $(D B)$ by

$\left(h_{0}(x), h_{1}(x)\right)=\left(\frac{-4 c \lambda}{a} \arctan \left(\frac{\varepsilon \sin (N x)}{1-\varepsilon \cos (N x)}\right), \frac{4 c^{2} \lambda N}{a} \frac{\varepsilon \sin (N x)}{1-2 \varepsilon \cos (N x)+\varepsilon^{2}}\right)$.

Hence we defone the following four families of initial data,

$$
\Theta_{i}^{ \pm}\left(\varepsilon_{0}\right)=\left\{\left(h_{0 i}^{ \pm}(x), h_{1 i}^{ \pm}(x)\right) / N>1,0<|\varepsilon| \leq \varepsilon_{0}<1\right\}, i=1,2 .
$$


Then we state non-existence theorem of $(D B)$.

Theorem 2.1. Let initial data $\left(h_{0}(x), h_{1}(x)\right) \in \Theta_{1}^{-}\left(\varepsilon_{0}\right)$. Then there exists $T>0$ such that $v(x, t)$ is the solution of $(D B)$ for $0<t<T$, and $v_{t}(x, t)$ and $v_{x}(x, t)$ blow up in finite time $t=T$ at some point $x \in[0, \pi]$.

Proof. First of all we show that $v(x, t)$ is a solution of $(D B)$ for $0 \leq t<T_{1}^{-}=-\log |\varepsilon| /\left(N c_{1}^{-}\right)$. From (2.9) it follows that for $v_{I}:=v_{I}(x, t)=$ $\sum_{n=1}^{I} a_{n} \exp (c N n t) \sin (N n x)$ and any test function $\varphi:=\varphi(x, t) \in \mathfrak{D}((0, \pi) \times$ $(0, T))$ we have

$$
\begin{gathered}
\left(v_{I t t}-\lambda v_{I x x t}-d v_{I x x}-\left(a v_{I x} v_{I x x}-\sum_{k=1}^{I-2} g_{k}(t) k \sin (N k x)\right), \varphi\right)= \\
\left(v_{I}, \varphi_{t t}\right)-\lambda\left(v_{I t}, \varphi_{x x}\right)-d\left(v_{I}, \varphi_{x x}\right)+\left(\frac{a}{2} v_{I x}^{2}+\sum_{k=1}^{I-2} g_{k}(t) N^{-1} \cos (N k x), \varphi_{x}\right)
\end{gathered}
$$

by Lemma 3 ii)

$$
\rightarrow\left(v_{t t}-\lambda v_{x x t}-d v_{x x}-\left(a v_{x}-\frac{N \lambda c(\varepsilon \exp (N c t))^{2}}{1-(\varepsilon \exp (N c t))^{2}}\right) v_{x x}, \varphi\right)=0
$$

as $I \rightarrow \infty$. Thus by $(2.25)$ we see that $v(x, t)$ is a solution of $(D B)$ for initial data $\left(h_{0}(x), h_{1}(x)\right) \in \Theta_{1}^{-}\left(\varepsilon_{0}\right)$.

$\mathbf{I}\left(\right.$ Blow-up of $\left.v_{x}(x, t)\right)$. In the case of $\cos \left(N x_{0}\right)=\operatorname{sgn}(\varepsilon)$, blow-up occurs in (2.18), since from (2.21) it follows that

$$
v_{x}\left(x_{0}, t\right)=\frac{-4}{a} N|\varepsilon| c_{1}^{-} \lambda \exp \left(N c_{1}^{-} t\right) \frac{1}{1-|\varepsilon| e^{N c_{1}^{-} t}} \rightarrow+\infty
$$

as $t \rightarrow T_{1}^{-}=-\log |\varepsilon| /\left(N c_{1}^{-}\right)$. Hence we see that $v_{x}(x, t)$ blows up at $\left(x_{0}, T_{1}^{-}\right)$. II(Blow-up of $\left.v_{t}(x, t)\right)$. Next we consider about the asymptotic behavior of $v_{t}(x, t)$. We see that $v_{t}\left(x_{0}+\delta, t\right)$ exists and possesses the same expression as $(2.19)$ in $\left[0, T_{1}^{-}\right]$for $0<|\delta|<\frac{\pi}{2}$. In fact, since for $0<|\delta|<\frac{\pi}{2 N}$ we have $0<\left|\cos \left(N\left(x_{0}+\delta\right)\right)\right|<1$, it holds that

$$
\left|\varepsilon \exp \left(N c_{1}^{-} t\right) \cos \left(N\left(x_{0}+\delta\right)\right)\right|<1, \quad \text { for } 0 \leq t \leq T_{1}^{-},
$$

which gurantees the convergence of $v_{t}\left(x_{0}+\delta, t\right)$ in $\left[0, T_{1}^{-}\right]$and for $t \leq T_{1}^{-}$it is represented by

$$
v_{t}\left(x_{0}+\delta, t\right)=\frac{-4 N c_{1}^{-2} \lambda \varepsilon}{a} \exp \left(N c_{1}^{-} t\right) \frac{\sin \left(N\left(x_{0}+\delta\right)\right)}{1-2 \varepsilon \exp \left(N c_{1}^{-} t\right) \cos \left(N\left(x_{0}+\delta\right)\right)+\varepsilon^{2} \exp \left(2 N c_{1}^{-} t\right)} .
$$


On the other hand we have for $\varepsilon>0$

$$
\begin{gathered}
1-2 \varepsilon \exp \left(N c_{1}^{-} T_{1}^{-}\right) \cos \left(N\left(x_{0}+\delta\right)\right)+\varepsilon^{2} \exp \left(2 N c_{1}^{-} T_{1}^{-}\right) \\
=2-2 \cos \left(N\left(x_{0}+\delta\right)\right)=4 \sin ^{2}\left(N\left(x_{0}+\delta\right) / 2\right)
\end{gathered}
$$

and for $\varepsilon<0$

$1-2 \varepsilon \exp \left(N c_{1}^{-} T_{1}^{-}\right) \cos \left(N\left(x_{0}+\delta\right)\right)+\varepsilon^{2} \exp \left(2 N c_{1}^{-} T_{1}^{-}\right)=4 \cos ^{2}\left(N\left(x_{0}+\delta\right) / 2\right)$.

Hence it follows that for $\varepsilon>0$

$$
\begin{gathered}
v_{t}\left(x_{0}+\delta, T_{1}^{-}\right)=\frac{-4}{a} N c_{1}^{-2} \lambda \varepsilon \exp \left(N c_{1}^{-} T_{1}^{-}\right) \frac{2 \sin \left(N\left(x_{0}+\delta\right) / 2\right) \cos \left(N\left(x_{0}+\delta\right) / 2\right)}{4 \sin ^{2}\left(N\left(x_{0}+\delta\right) / 2\right)} \\
=-\frac{2}{a} N c_{1}^{-2} \lambda \varepsilon \exp \left(N c_{1}^{-} T_{1}^{-}\right) \frac{\cos \left(N\left(x_{0}+\delta\right) / 2\right)}{\sin \left(N\left(x_{0}+\delta\right) / 2\right)}
\end{gathered}
$$

and that for $\varepsilon<0$

$$
v_{t}\left(x_{0}+\delta, T_{1}^{-}\right)=-\frac{2 N c_{1}^{-2} \lambda \varepsilon}{a} \exp \left(N c_{1}^{-} T_{1}^{-}\right) \frac{\sin \left(N\left(x_{0}+\delta\right) / 2\right)}{\cos \left(N\left(x_{0}+\delta\right) / 2\right)} .
$$

Considering into $\operatorname{sgn}(\varepsilon)=\cos \left(N x_{0}\right)=2 \cos ^{2}\left(N x_{0} / 2\right)-1=1-2 \sin ^{2}\left(N x_{0} / 2\right)$, we have

$$
\cos ^{2}\left(N x_{0} / 2\right)=\frac{1+\operatorname{sgn}(\varepsilon)}{2} \text { and } \sin ^{2}\left(N x_{0} / 2\right)=\frac{1-\operatorname{sgn}(\varepsilon)}{2} .
$$

Since in the case of $\varepsilon>0(2.28)$ implies that for sufficiently small $|\delta|$

$$
\left|\cos \left(N\left(x_{0}+\delta\right) / 2\right)\right|>\frac{1}{2},
$$

we have by (2.26) and (2.28)

$$
\lim _{\delta \rightarrow 0}\left|v_{t}\left(x_{0}+\delta, T_{1}^{-}\right)\right| \geq \frac{N \lambda c_{1}^{-2}}{a} \lim _{\delta \rightarrow 0} \frac{1}{\left|\sin \left(N\left(x_{0}+\delta\right) / 2\right)\right|}=\infty .
$$

When $\varepsilon<0$, by the same manner as above and using (2.27) and (2.28) we have

$$
\lim _{\delta \rightarrow 0}\left|v_{t}\left(x_{0}+\delta, T_{1}^{-}\right)\right| \geq \frac{N \lambda c_{1}^{-2}}{a} \lim _{\delta \rightarrow 0} \frac{1}{\left|\cos \left(N\left(x_{0}+\delta\right) / 2\right)\right|}=\infty .
$$


Consequently for any constant $L>0$, there exists $\delta_{0}>0$ such that for $0<|\delta|<\delta_{0}$

$$
\lim _{t \rightarrow T_{1}^{-}}\left|v_{t}\left(x_{0}+\delta, t\right)\right| \geq L
$$

which means that we find a blow-up point of $v_{t}(x, t)$ at $\left(x_{0}, T_{1}^{-}\right)$in such a sense. Hence the proof is complete.

In the case of $c=c_{2}^{-}<0$, in the same way as above we obtain another solution of $(D B)$ for $d<0$

$$
v(x, t)=\frac{-4 \lambda c_{2}^{-}}{a} \sum_{n=1}^{\infty} \frac{\varepsilon^{n} \exp \left(c_{2}^{-} N n t\right)}{n} \sin (N n x) .
$$

In the same argument as used in the proof of lemma 3 there exists a constant $1>\varepsilon_{0}>0$ such that $v(x, t)$ converges for any $0<|\varepsilon| \leq \varepsilon_{0}$ and we obtain the same type of representations of

$$
v(x, t)=\frac{-4 \lambda c_{2}^{-}}{a} \arctan \left(\frac{\varepsilon \exp \left(c_{2}^{-} N t\right) \sin (N x)}{1-\varepsilon \exp \left(c_{2}^{-} N t\right) \cos (N x)}\right)
$$

and its derivatives with respect to $x$ and $t$ as (2.18) and (2.19) respectively for $c=c_{2}^{-}<0$ and $(x, t) \in(0, \pi) \times(0, \infty)$. Then we have the following result.

Theorem 2.2. For initial data $\left(h_{0}(x), h_{1}(x)\right) \in \Theta_{2}^{-}\left(\varepsilon_{0}\right)$ there exists a solution $v(x, t)$ of $(D B)$ for $0<t$ such that it holds that for a constant $C>0$

$$
\left|\partial_{t}^{i} \partial_{x}^{j} v(x, t)\right| \leq C e^{N c_{2}^{-} t}, 0 \leq i+j \leq 1
$$

Proof. For $|\varepsilon|<1$ we see

$$
1-2 \varepsilon \exp \left(N c_{2}^{-} t\right) \cos (N x)+\varepsilon^{2} \exp \left(2 N c_{2}^{-} t\right)>\left(1-\varepsilon \exp \left(N c_{2}^{-} t\right)\right)^{2}>0
$$

for any $t>0$. Hence the representations of $v(x, t), v_{x}(x, t)$ and $v_{t}(x, t)$ show that they decays exponentially as $t \rightarrow \infty$ and that (2.30) holds.

In the same way as in the proof of theorem 2.2 we have the following result.

Theorem 2.3. For initial data $\left(h_{0}(x), h_{1}(x)\right) \in \Theta_{1}^{+}\left(\varepsilon_{0}\right)$ or $\Theta_{2}^{+}\left(\varepsilon_{0}\right)$, there exists a solution $v(x, t)$ of $(D B)$ for $t>0$ such that it holds for a constant $C>0$

$$
\sum_{i+j \leq 1}\left|\partial_{t}^{i} \partial_{x}^{j} v(x, t)\right| \leq C e^{N c_{i}^{+} t}, i=1,2 .
$$


Proof. By the analogous way to the proof of Theorem 2.2 we obtain the decay estimate $(2.31)$ considering into $c_{i}^{+}<0, i=1,2$. Hence we omit the proof.

\subsection{Existence and non-existence of global solutions to (GE)}

In this subsection, we discuss existence and non-existence of global solutions of a generalized problem of $(G E)$ :

$(G E)_{p}\left\{\begin{array}{l}u_{t t}-\lambda u_{x x t}=A_{p}\left(u_{x}\right)=\left(a u_{x}+\sigma\left(u_{x}-\frac{p}{\pi}\right)\right) u_{x x}, \quad(0, \pi) \times(0, T) \\ u(0, t)=0, u(\pi, t)=p \\ u(x, 0)=h_{0}(x)+\frac{p}{\pi} x, u_{t}(x, 0)=h_{1}(x) .\end{array}\right.$ where $p$ is a real number. In fact, putting $p=\pi, h_{0}(x) \equiv 0$ and $h_{1}(x) \equiv$ $V^{0}(x),(G E)_{p}$ coincides with $(G E)$. By setting $u(x, t)=p x / \pi+v(x, t),(G E)_{p}$ is reduced to

$(G E)_{p}^{\prime}\left\{\begin{array}{l}v_{t t}-\lambda v_{x x t}-\frac{p a}{\pi} v_{x x}=\left(a v_{x}+\sigma\left(v_{x}\right)\right) v_{x x} \\ v(0, t)=0, v(\pi, t)=0 \\ v(x, 0)=h_{0}(x), v_{t}(x, 0)=h_{1}(x) .\end{array}\right.$

Since $(G E)_{p}^{\prime}$ is the same type of the problem as $(D B)$, by theorems 2.1-2.3 we obtain the following result.

Corollary 2.1. i) In the case of $p a<0$, for intial data given in theorem 2.1 there exists the solution $v(x, t)$ of $(G E)_{p}^{\prime}$ in $0<t<T_{1}^{-}$such that $u_{x}(x, t)$ and $u_{t}(x, t)$ blow up at $t=T_{1}^{-}$for the solution $u(x, t)=p x / \pi+v(x, t)$ of $(G E)_{p}$ and some points $x \in[0, \pi]$. For intial data given in theorem 2.2 there exits the global solution $v(x, t)$ of $(G E)_{p}^{\prime}$ such that $v(x, t)$ satisfies for a positive constant $k$

$$
v(x, t)=o\left(e^{-k t}\right),
$$

and the solution $u(x, t)=p x / \pi+v(x, t)$ of $(G E)_{p}$ satisfies

$$
\lim _{t \rightarrow \infty} u_{x}(x, t)=\frac{p}{\pi}
$$

ii) In the case of $p a>0$, for intial data given in theorem 2.3 there exists the global solution $v(x, t)$ of $(G E)_{p}^{\prime}$ such that $v(x, t)$ satisfies $v(x, t)=o\left(e^{-k t}\right)$ 
for a positive constant $k$ and the solution $u(x, t)=p x / \pi+v(x, t)$ of $(G E)_{p}$ satisfies (2.32).

By corollary 2.1 we discuss the relationship between the property of $A_{p}\left(u_{x}\right)$ and existence and non-existence of the solutions comparing with conditions (1.6) and (1.8) given by Greenberg and Ebihara respectively. For the simplicity, $p$ is assumed to be positive below.

1) If such conditions are not satisfied, generally there do not exist global solutions to the problems. In fact, since by corollary $2.1 \mathrm{i}$ ) we have

$$
v_{x}\left(x_{0}, t\right)>0 \text { and } \lim _{t \rightarrow T_{1}^{-}-0} v_{x}\left(x_{0}, t\right)=+\infty
$$

for the solution $v(x, t)$ of $(G E)_{p}^{\prime}$ with $a<0$ and $\left(h_{0}(x), h_{1}(x)\right) \in \Theta_{1}^{-}\left(\varepsilon_{0}\right)$, taking account of Remark, it is seen that

$$
A_{p}\left(u_{x}\right)=a\left(\frac{p}{\pi}+v_{x}\right)+\sigma\left(v_{x}\right)<0 \rightarrow-\infty \text { as } t \rightarrow T_{1}^{-}-0
$$

which does not necessarily satisfy (1.6) and (1.8).

2) On the other hand, corollary 2.1 i) also implies that there exist time global solutions $u(x, t)$ of $(G E)_{p}^{\prime}$ for $a<0$ and $\left(h_{0}(x), h_{1}(x)\right) \in \Theta_{2}^{-}\left(\varepsilon_{0}\right)$ even if (1.6) and (1.8) do not hold for $A_{p}\left(u_{x}\right)$. In fact in this case it is seen that for sufficiently large $T>0$ we have by the expression of $\sigma\left(v_{x}\right)$ in (2.17),

$$
A_{p}\left(u_{x}\right)=a\left(\frac{p}{\pi}+v_{x}\right)+\sigma\left(v_{x}\right)<0, t \geq T
$$

taking account of Remark and $v(x, t)=o\left(e^{-k t}\right)$.

3) Next, since the solution $u(x, t)$ to $(G E)_{p}$ for $a>0$ and $\left(h_{0}(x), h_{1}(x)\right) \in$ $\Theta_{i}^{+}\left(\varepsilon_{0}\right), i=1,2$, possesses the same asymptotic property as $(2.32)$, considering into Remark we see that

$$
A_{p}\left(u_{x}\right)=a\left(\frac{p}{\pi}+v_{x}\right)+\sigma\left(v_{x}\right)>0, \quad t \geq T
$$

holds for sufficiently large $T>0$ in $(G E)_{p}$. Consequently our result about $(G E)_{p}$ for $a<0$ is a counter part of results of Greenberg [5] and Ebihara [3] and for $a>0$ it is corrsponding to their results.

\section{Acknowledgement}


This work is partially supported by Les fonctions d'enseignante invité No. 2006-179, École Centrale de Lyon, Grant-in-Aid for Scientific Research (C) 16540176 and 19540200 from Japan Society for the Promotion of Science. One of the authors would like to express one's sincere gratitude to the collegues of École Centrale de Lyon for their hospitality.

\section{References}

[1] B. Davis, Reinforced random walks, Probability Theory and Related Fields, 84, 1990, 203-229.

[2] Y. Ebihara, On some nonlinear evolution equations with the strong dissipation, J. Differential Equations, 30,1978, 149-164.

[3] Y. Ebihara, On some nonlinear evolution equations with the strong dissipation, II, J. Differential Equations, 34, 1979, 339-352.

[4] Y. Ebihara, On some nonlinear evolution equations with the strong dissipation, III, J. Differential Equations, 45, 1982, 332-355.

[5] J.M. Greenberg, On the existence, uniqueness, and stability of solutions of the equation $\rho_{0} X_{t t}=E\left(X_{x}\right) X_{x x}+\lambda X_{x x t}$, J. Math. Anal. Appl., 25, $1969,575-591$.

[6] J.M. Greenberg, R. MacCamy and V. Mizel, On the existence, uniqueness, and stability of solutions of the equation $\sigma^{\prime}\left(u_{x}\right) u_{x x}+\lambda u_{x t x}=\rho u_{t t}$, J. Math. Mech., 17, 1968, 707-728.

[7] T. Kakita, Time periodic solutions of some nonlinear evolution equations, Publ. RIMS, Kyoto Univ., 9, 1974, 477-492.

[8] S. Kawashima and Y. Shibata, Global existence and exponential decay of small solutions to nolinear viscoelasticity, Commun. Math. Phys. , 148, 1992, 189-208.

[9] T. Kobayashi, H. Pecher, and Y. Shibata, On a global in time existence theorem of smooth solutions to nonlinear wave equation with viscosity, Math. Ann., 296, 1993, 215-234. 
[10] H.A. Levine and B.D. Sleeman, A system of reaction and diffusion equations arising in the theory of reinforced random walks, SIAM J. Appl. Math., 57-3,1997, 683-730.

[11] B.D. Sleeman and H.A. Levine, Partial differential equations of chemotaxis and angiogenesis, Math. Mech. Appl. Sci., 24, 2001, 405-426.

[12] H.G. Othmer and A. Stevens, Aggregation, blowup, and collapse: The ABC's of taxis in reinforced random walks, SIAM J. Appl. Math., 574,1997, 1044-1081.

[13] M. Tsutumi, Some nonlinear evolution equations of second order, Proc. Japan Acad., 47, 1971, 950-955.

[14] Y. Yang, H. Chen and W. Liu, On existence and non-existence of global solutions to a system of reaction- diffusion euations modeling chemotaxis, SIAM J. Math. Anal., 33, 1997, 736-785.

[15] Y. Yamada, Some remarks on the equation $y_{t t}-\sigma\left(y_{x}\right) y_{x x}-y_{x x t}=f$, Osaka J. Math., 17, 1980, 303-323. 